\title{
Serum metabonomics as a diagnostic approach for cancer-related fatigue
}

\author{
HAIMING WANG ${ }^{1,2^{*}}$, TONG ZHAO ${ }^{1,2^{*}}, \mathrm{CAN} \mathrm{LV}^{2}, \mathrm{ZHAN} \mathrm{ZHANG}^{2}, \mathrm{FANFU} \mathrm{FANG}^{2}$ and BAI $\mathrm{LI}^{2}$ \\ ${ }^{1}$ Department of Integrated Chinese and Western Medicine, School of Chinese Medicine \& School of Integrated Chinese \\ and Western Medicine, Nanjing University of Chinese Medicine, Nanjing, Jiangsu 210023; ${ }^{2}$ Department of Rehabilitation \\ Medicine, Changhai Hospital of Shanghai, The Second Military Medical University, Shanghai 200433, P.R. China
}

Received January 4, 2020; Accepted February 3, 2021

DOI: $10.3892 /$ etm.2022.11181

\begin{abstract}
In the present study, differences in metabolic pathways between patients with and without cancer-related fatigue (CRF) were examined to identify metabolic serum biomarkers of CRF. In this preliminary study, metabolic profiling was applied to analyze the serum samples from 14 patients with CRF and 11 non-CRF individuals (non-fatigue cancer survivors) by ultra-performance liquid chromatography coupled with mass spectrometry. Orthogonal partial least-squares discriminant analysis was adopted to evaluate the differences between the CRF and non-CRF groups. The CRF group was characterized by increases in phosphatidylethanolamine (PE; 18:0/0:0), LysoPE (0:0/20:4 and 0:0/16:0), lysophosphatidylcholine (LysoPC; 20:4, 22:4 and 16:0) and LysoPC/PC, phosphatidylserine (21:0/0:0), glycerophosphocholine and N-docosahexaenoyl $\gamma$-aminobutyric acid. Furthermore, decreases in anandamide, uric acid, dihydrouracil, LysoPE (0:0/22:5), 2,5,7,8-tetramethyl-2(2'-carboxyethyl)-6-hydroxychroman,19(R)hydroxy-prostaglandin $\mathrm{F} 1 \alpha, \mathrm{N}-(3 \alpha, 12 \alpha$-dihydroxy-5 $\beta$ cholan-24-oyl)-glycine, ketoleucine, indoxyl sulfate, $\alpha-\mathrm{N}$-phenylacetyl-L-glutamine and 1-linoleoyl-glycerophosphocholine were detected. These data indicate a possible disturbance in the metabolism of phospholipids and adjustments in the endocannabinoid system. The metabonomic approach may be helpful to determine the pathophysiological mechanisms of CRF and the identification of potential biomarkers for the accurate diagnosis of CRF. All clinical
\end{abstract}

Correspondence to: Dr Bai Li or Dr Fanfu Fang, Department of Rehabilitation Medicine, Changhai Hospital of Shanghai, The Second Military Medical University, 800 Xiangyin Road, Shanghai 200433, P.R. China

E-mail: 1ibai9@126.com

E-mail: fangfanfu@126.com

${ }^{*}$ Contributed equally

Key words: metabolic profiling, UPLC/MS, diagnostic approach, cancer-related fatigue, serum data were obtained from the 'Research on the efficacy of traditional Chinese medicine comprehensive intervention in cancer-related fatigue' (TCM-CRF) project. Medical Ethical Approval for TCM-CRF was approved by the Chinese Ethics Committee of Registering Clinical Trials. The approval number for the TCM-CRF study was ChiECRCT-2013038, and the TCM-CRF study was completed.

\section{Introduction}

Cancer-related fatigue (CRF) is one of the most troubling symptoms in patients with cancer. It was reported in 1979 by Haylock and Hart (1) for the first time. The National Comprehensive Cancer Network (NCCN) guidelines for CRF (version II; 2018) (2) define it as a distressing, persistent and subjective sense of physical, emotional and/or cognitive tiredness or exhaustion that is related to cancer treatment. The fatigue is not related to recent activity and it interferes with usual functioning. According to surveys, up to $90 \%$ of cancer patients suffer from various degrees of CRF $(2,3)$. However, due to an insufficient understanding and lack of effective interventions, fatigue makes patients feel more uncomfortable compared with symptoms such as pain and vomiting, as the latter may be treated $(4,5)$. Certain cancer survivors may experience fatigue for long periods of time and in numerous cases, may go on for 5-10 years $(6,7)$.

The pathophysiological mechanisms of CRF have remained to be fully elucidated. Previous studies focused on the effects of chronic inflammation and neuroendocrine factors on CRF, suggesting that the activation of proinflammatory cytokines drives CRF.

A number of clinical studies have indicated that fatigue is closely related to plasma IL-6 levels $(8,9)$. Orre et al $(10)$ proposed that the levels of circulating IL-1 receptor antagonist and C-reactive protein in long-term survivors of testicular cancer with chronic CRF were higher than those in fatigue-free patients. Another study revealed that cortisol levels were low in breast cancer survivors with CRF (11). However, most studies tested certain target molecules thought to be involved in the pathological mechanisms underlying $\mathrm{CRF}$, while broad-spectrum analysis had remained to be performed.

In the present study, comprehensive profiling of the serum metabolomes was performed for patients with CRF 
to screen for pathways and metabolic systems possibly related to the development of CRF. As an important component of systems biology, metabonomics is a subcategory of metabolomics. It complements genomics and proteomics by analyzing global metabolic responses to pathological stimuli in tissues and extracellular biological fluids, including urine and blood (12). Metabonomic analyses such as that of the present study will provide novel insight into the global effects of diseases on metabolic pathways. Ultra-performance liquid chromatography coupled to mass spectrometry (UPLC/MS) is considered a powerful analytical tool that is increasingly applied to metabonomic profiling (13). In the present study, UPLC/MS was adopted to examine the differences in metabolic pathways of patients with and without CRF to identify metabolic biomarkers of CRF in blood. The present results provided evidence that the UPLC/MS may be utilized as a tool for diagnosing and evaluating CRF.

\section{Materials and methods}

Patients. Outpatients receiving medical care at the Department of Traditional Chinese Medicine of Changhai Hospital, The First Affiliated Hospital of the Second Military Medical University (Shanghai, China) between February 2014 and November 2014 were recruited for the present study. The inclusion criteria were as follows: i) Adult outpatients with stage IIIA/IIIB colorectal cancer (as classified in the 7 th edition of the American Joint Committee on Cancer Staging Manual and the Future of TNM) (14) after radical surgery (colectomy with enbloc removal of regional lymph nodes) and standard chemotherapy with mFOLFOX6 [oxaliplatin $85 \mathrm{mg} / \mathrm{m}^{2}$ intravenously (IV) over $2 \mathrm{~h}$, day one; leucovorin $400 \mathrm{mg} / \mathrm{m}^{2} \mathrm{IV}$ over $2 \mathrm{~h}$, day one; 5 -fluorouracil $400 \mathrm{mg} / \mathrm{m}^{2} \mathrm{IV}$ bolus on day one, then $1,200 \mathrm{mg} / \mathrm{m}^{2} /$ day $\mathrm{x} 2$ days (total $2,400 \mathrm{mg} / \mathrm{m}^{2}$ over $46-48 \mathrm{~h}$ ) IV continuous infusion and repeat every two weeks for 12 cycles]; ii) 2 months to 2 years after radical surgery and adjuvant chemotherapy; iii) no indications of tumor extension or new metastasis during follow-up (history, physical examination, carcinoembryonic antigen and chest/abdominal/pelvic CT every three months); iv) the patient's symptoms meet the diagnostic standards in the NCCN practice guidelines for CRF (version I/2012) (15); v) participants completed the Piper Fatigue Scale-Revised, CRF patients with a history of CRF [score $\geq 3.4$ Piper fatigue scale (16)]; vi) written informed consent; vii) CRF group's fatigue symptoms appeared during and after adjuvant chemotherapy and continued throughout the follow-up process.

The following exclusion criteria were applied: i) Non cancer-related causes of fatigue, such as uncontrolled pain, insomnia or hypothyroidism; ii) other diseases, including diabetes mellitus and hyperthyroidism, or drug treatment that is able to interfere with the metabolism; iii) radiotherapy or chemotherapy within four weeks prior to recruitment.

After screening 70 patients, $15 \mathrm{CRF}$ patients and 15 non-CRF patients were enrolled. According to the Piper fatigue scale (16), patients in the non-CRF group were fatigue-free. Prior to collecting blood, two patients in the non-CRF group were excluded as their high blood glucose levels indicated an insulin-related disorder. The final cohort included $15 \mathrm{CRF}$ patients and 13 non-CRF subjects.

All clinical data in the present study were obtained from the 'Research on the efficacy of TCM comprehensive intervention in cancer-related fatigue' (TCM-CRF) project. Medical Ethical Approval for TCM-CRF was approved by the Chinese Ethics Committee of Registering Clinical Trials (ChiERCT) at West China Hospital (Chengdu, China). The approval number for the TCM-CRF study was ChiECRCT-2013038, and the TCM-CRF study was completed. The patients' characteristics are provided in Table I.

Sample collection and preparation. The blood samples were collected into BD Vacutainer ${ }^{\circledR}$ blood collection tubes (BD SST; BD Biosciences) by venipuncture. Prior to sampling, no treatment had been given to the patients. They were requested to consume a normal diet and to avoid overeating, smoking, drinking and performing any strenuous exercise. All blood samples were taken at the same time (7:00-8:00 a.m.) on an empty stomach. Routine blood parameters and liver function were checked at the same time. All blood samples were stored in a refrigerator for 30 min prior to being transferred to the College of Pharmacy of the Second Military Medical University (Shanghai, China). The serum was separated from the clotted whole blood by centrifugation at $1,500 \mathrm{x}$ g for $10 \mathrm{~min}$. A $500 \mu \mathrm{l}$ serum aliquot was collected for each patient and stored at $-80^{\circ} \mathrm{C}$ until analysis.

Prior to analysis, all 28 serum samples were simultaneously thawed at room temperature and a $100 \mu \mathrm{l}$ aliquot was mixed with ice-cold deuterated chloroform and methanol (300 $\mu \mathrm{l}$ each) by vortexing, and the mixture was then left on ice for $10 \mathrm{~min}$. Subsequently, samples were centrifuged at $16,000 \mathrm{x} \mathrm{g}$ for $15 \mathrm{~min}$ at $4^{\circ} \mathrm{C}$ to separate hydrophilic and lipophilic phases of water/methanol and chloroform, respectively.

UPLC/MS analysis of serum samples. The metabolic profiling analysis of serum was performed for all samples by using a Waters ACQUITY UPLC system (Waters Corp.) combined with an Agilent 6538 Ultra High Definition (UHD) and Accurate-Mass Quadrupole time-of-flight (Q-TOF)/mass spectrometer (MS) (Waters Corp.). Chromatographic separation was performed on an ACQUITY UPLC@HSS T3 column (100 ̊, $1.8 \mu \mathrm{m}, 2.1 \mathrm{~mm}$; Waters Corp.) at $40^{\circ} \mathrm{C}$. The mobile phase consisted of $0.1 \%$ formic acid in water (component $\mathrm{A}$ ) and $0.1 \%$ formic acid in acetonitrile (component $\mathrm{B}$ ) at a flow rate of $350 \mu \mathrm{l} / \mathrm{min}$. The column was eluted with a linear gradient of $5 \%$ B for $0-1 \mathrm{~min}, 5-95 \%$ B for 1-10 min and $95 \%$ B for $10-12$ min. Furthermore, $95 \% \mathrm{~B}$ was held for $1 \mathrm{~min}$ after elution and then changed to $5 \% \mathrm{~B}$ for an additional $5 \mathrm{~min}$ to re-equilibrate the column before injecting the next sample. All samples were maintained at $4^{\circ} \mathrm{C}$ throughout the analysis. MS data were obtained by using Agilent 6538 UHD and Accurate-Mass Q-TOF/MS systems (Waters Corp.) equipped with an electrospray source and operating in both positive and negative ion modes. The parameters were as follows: Gas temperature, $350^{\circ} \mathrm{C}$; gas flow, $11 \mathrm{l} / \mathrm{min}$; nebulizer, $45 \mathrm{psi}$; fragmentor, $120 \mathrm{~V}$; skimmer, CK160V; octopole radio frequency (RF) peak, $750 \mathrm{~V}$. Reference mass correction: Mass-to-charge ratio (m/z) 121.0509 and 922.0098 for positive mode, Capillary Voltage (VCap): 4,000; m/z 112.985587 and 1,033.988109 for negative mode, VCap: 3,000. 
Table I. Patients' characteristics.

\begin{tabular}{|c|c|c|c|}
\hline Parameters & CRF group $(n=15)$ & non-CRF group $(n=13)$ & P-value \\
\hline Sex & & & 0.274 \\
\hline Male & $5(33.3)$ & $7(53.8)$ & \\
\hline Female & $10(66.7)$ & $6(46.2)$ & \\
\hline Age, years & $53 \pm 19$ & $53 \pm 13$ & 0.93 \\
\hline $\mathrm{RBC}, 10^{12} / 1$ & $4.16 \pm 0.44$ & $4.45 \pm 0.62$ & 0.11 \\
\hline $\mathrm{HGB}, \mathrm{g} / 1$ & $125.00 \pm 23.00$ & $134.00 \pm 20.00$ & 0.22 \\
\hline $\mathrm{WBC}, 10^{9} / 1$ & $4.90 \pm 1.50$ & $5.07 \pm 1.14$ & 0.55 \\
\hline ALT, U/l & $22.00 \pm 7.00$ & $24 \pm 13.50$ & 0.68 \\
\hline $\mathrm{AST}, \mathrm{U} / 1$ & $20.00 \pm 10.00$ & $20 \pm 16.50$ & 0.34 \\
\hline
\end{tabular}

Values are expressed as $\mathrm{n}(\%)$ or the mean \pm standard deviation. CRF, cancer-related fatigue; RBC, red blood cells; HGB, hemoglobin; WBC, white blood cells; ALT, alanine transaminase; AST, aspartate transaminase.

Data analysis. After screening the preliminary data, any abnormal data in the CRF group and non-CRF group were excluded ( 1 case and 2 cases, respectively). Finally, 14 samples were confirmed for the CRF group and 11 samples for the non-CRF group.

All clinical data were tested for normal distribution and similarity of variance. Differences between two groups were analyzed by one-way ANOVA if the data were normally distributed and the variances were homogenous. The measurement data of non-normal distribution were tested by rank transformation non-parametric test. $\chi^{2}$ test was used for counting data. SPSS17.0 (SPSS, Inc.) was used for statistical analysis. The raw data were analyzed and processed with the Micromass Marker Lynx Application Manager (v.4.0; Waters Corp.). All data were normalized to the total ion intensity of each chromatogram to obtain the relative intensity of all metabolites. Three-dimensional data, including peak value (retention time and $\mathrm{m} / \mathrm{z}$ pairs), sample name and normalized ion intensity, were imported to SIMCA-P software (v.10.0; Umetrics) and orthogonal partial least-squares discriminant analysis (OPLS-DA) was performed. The permutation test was performed for the OPLS-DA model. Student's t-test (SPSS 17.0; SPSS, Inc.) was performed to evaluate the difference in sucrose consumption and $\mathrm{P}<0.05$ was considered to indicate a statistically significant difference. On the $\mathrm{R}$ software platform, the XCMS program (version 3.5) was used for peak extraction, alignment and deconvolution analysis. The exact molecular weights of differential metabolites were compared with entries in network databases, such as the Human Metabolome Database (HMDB; http://www. hmdb.ca), METLIN (http://metlin.scripps.edu) and Kyoto Encyclopedia of Genes and Genomes (KEGG; http://www. kegg.jp).

\section{Results}

Patients' characteristics. There was no statistically significant difference between the CRF and non-CRF groups in terms of sex $\left(P=0.274, \chi^{2}=1.197\right.$; Table I $)$. The age of the patients with CRF ranged from 44 to 68 years (median age, 53 years) and that of the non-CRF patients ranged from 44 to 69 years (median age, 53 years), and there was no statistically significant difference in age between the groups ( $\mathrm{P}=0.928$; Table I).

There were also no statistically significant differences between the two groups in terms of blood parameters $(\mathrm{P}>0.05)$, indicating that the interference of any abnormal factors, such as anemia and hepatic dysfunction, may be ruled out from the metabonomics study on CRF (Table I).

UPLC/MSmetabolic profiling. UPLC uses a separation medium for small particles and provides effective chromatographic separation of low-molecular-weight metabolites. Each serum sample was analyzed by applying both positive and negative MS ion modes. In Fig. 1, positive and negative ion base peak intensity chromatograms of serum samples from patients with CRF or non-CRF subjects are provided.

Multivariate analysis of UPLC/MS data. In order to identify potential biomarkers from a large amount of data from the ULPC/MS analysis, the multivariate pattern recognition method was adopted. OPLS-DA evaluates the difference between the samples in the two groups by Pareto scaling, orthogonal signal correction, filtering, category judgment using orthogonal uncorrelated variable information, category judgments of related variables and PLS-DA.

The R2Y value represents the goodness of fit of the model and the Q2 value refers to the predictability of the model. In the positive ion mode, $\mathrm{R} 2 \mathrm{Y}=0.963$ and $\mathrm{Q} 2=0.963$ were determined, and in the negative ion mode, $\mathrm{R} 2 \mathrm{Y}=0.823$ and $\mathrm{Q} 2=0.291$ were obtained. Clear separations between the CRF group and non-CRF group in both positive and negative ion modes were observed (Fig. 2), suggesting that biochemical changes occurred in the serum of patients with CRF.

In order to prevent the model from overfitting, the OPLS-DA model was tested by 200 times response permutation testing (RPT) (Fig. 2). The variables of a previously defined classification y matrix (such as 0 or 1 ) were randomly arranged $\mathrm{n}$ times $(\mathrm{n}=200)$. The corresponding OPLS-DA model was established to obtain R2 and Q2 values of the random model. Through linear regression with R2Y and Q2Y of the original model, the intercept values of the regression line and the $y$-axis were determined as R2 and Q2, respectively. The RPT test 


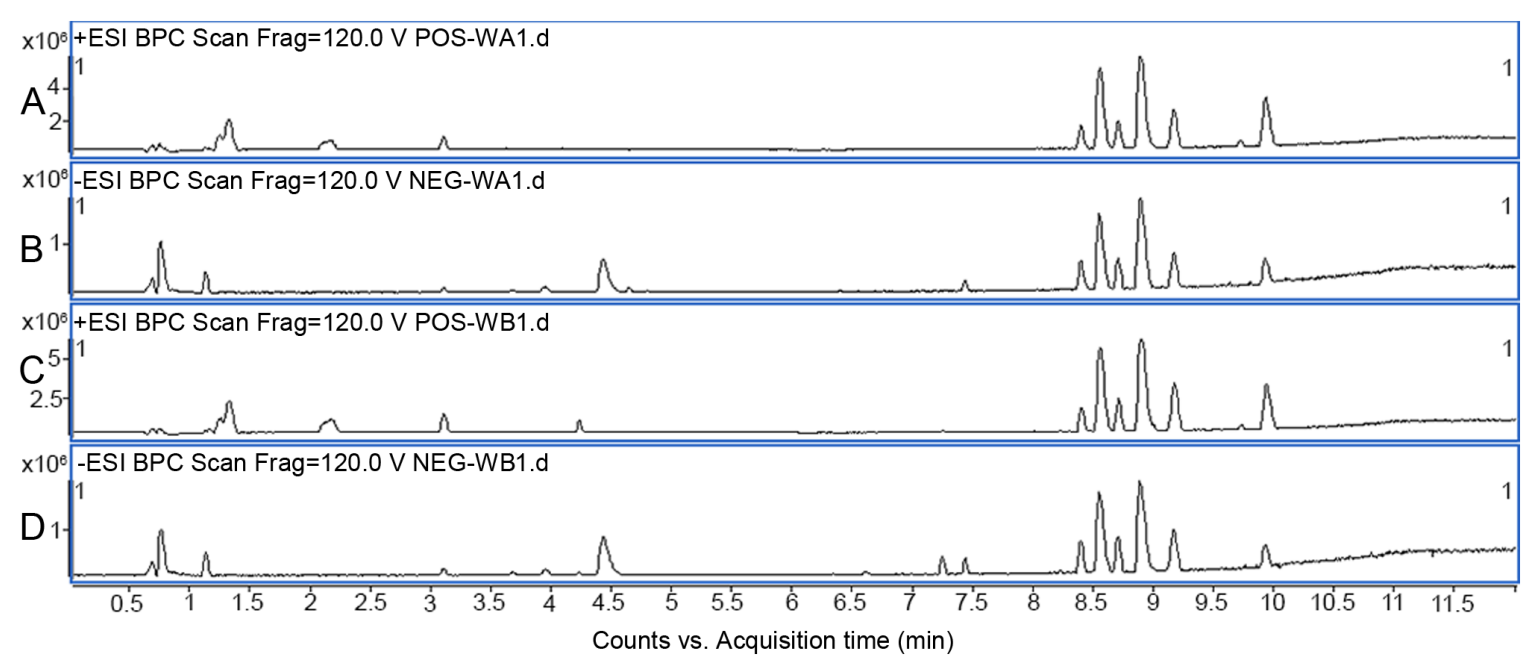

Figure 1. Positive and negative ion base peak intensity ( $\mathrm{mAu}$ ) chromatograms of representative serum samples from a patient with $\mathrm{CRF}$ (WA) and a non-CRF (WB) subject. (A and B) Patient with CRF in (A) positive and (B) negative mode; (C and D) non-CRF in (C) positive and (D) negative mode. CRF, cancer-related fatigue; ESI, electron spray ionization; BPC, Base Peak Chromatogram; neg, negative; pos, positive; Frag, fragmentation.
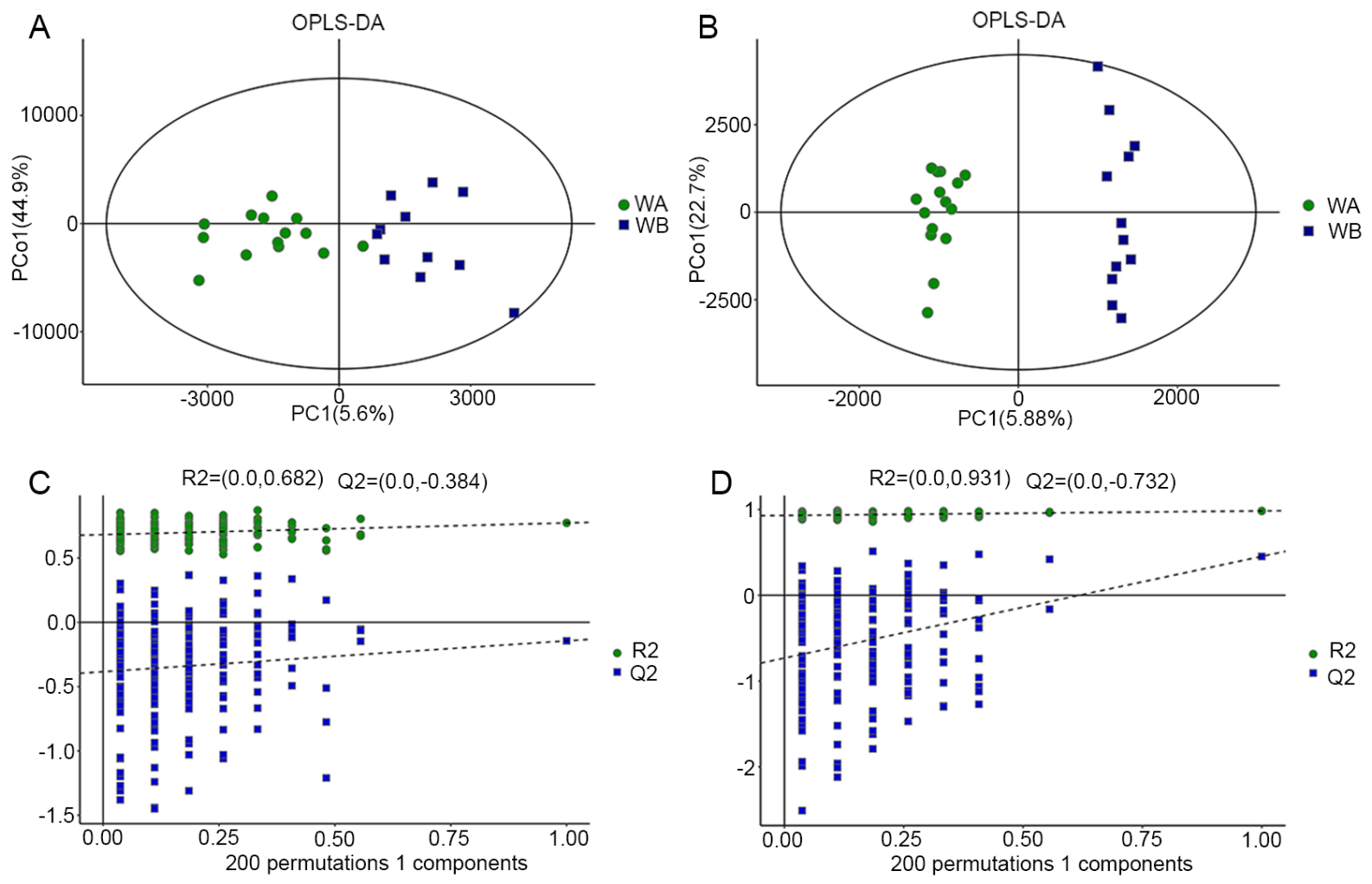

Figure 2. Scores in (A) positive and (B) negative ion modes from the OPLS-DA model classifying ultra-performance liquid chromatography tandem mass spectrometry data in CRF (WA, green squares) and non-CRF (WB, blue squares) patients. Permutation test of the OPLS-DA model of WA and WB in (C) positive and (D) negative ion mode. The R2 (cum) and Q2 (cum) are the two parameters used to describe the goodness of the model at the minimum number of components cumulatively required (cum) to optimally give account of the data variability. OPLS-DA, orthogonal partial least-squares discriminant analysis; PC, principal component.

revealed that Q2 was less than zero, which indicated that the model was not overfitting.

S-plots and VIP-value plots were combined for the visualization and screening of different metabolites. The red squares represent the VIP-value plot and the black triangles represent the S-plot (Fig. 3). In the present study, serum metabolites with a VIP value $>1.5$ and $\mathrm{P}<0.05$ were considered to be significantly different between the groups. Metabolite identification was based on the mass assignment and MS/MS ion analysis. As the sample pool was not very large, it was decided that the available online database resources, such as KEGG, METLIN and HMDB, would be used for comparisons.

The differential metabolites were then determined. Significant variables were detected in positive and negative ion 

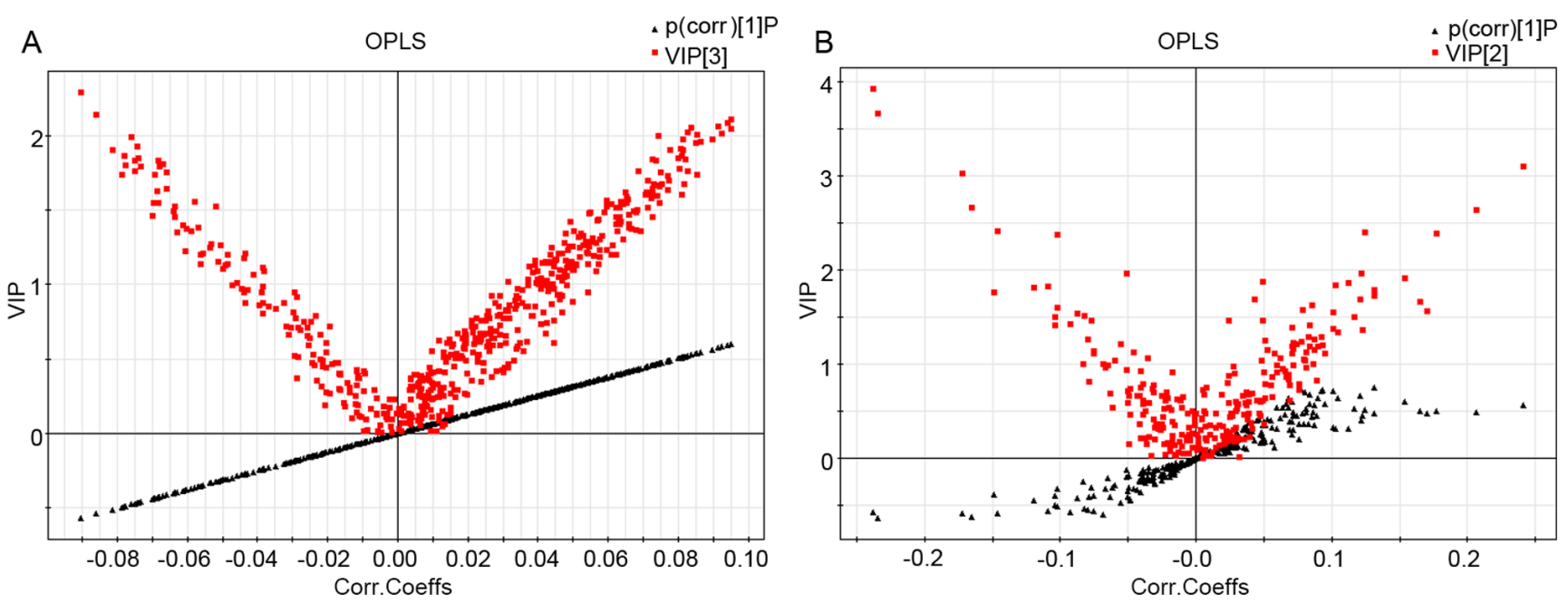

Figure 3. S-VIP plot in (A) positive and (B) negative ion modes from the OPLS-discriminant analysis model. The red squares represent the VIP-value plot. The black triangles represent the S-plot. OPLS, orthogonal partial least-squares; VIP, variable importance in the projection; p(corr), partial correlation coefficient; Corr.Coeffs, correlation coefficient.

modes and are summarized in Table II. Table II also outlines the results from the analysis, including the retention time, $\mathrm{m} / \mathrm{z}$, metabolite identification, variation tendency and statistical significance. The results were compared to the non-CRF group and significant changes in 21 metabolites were identified $(\mathrm{P}<0.05)$, such as the increase of phosphatidylethanolamine (PE; 18:0/0:0), LysoPE (0:0/20:4 and 0:0/16:0), phosphatidylserine (PS; 21:0/0:0) and lysophosphatidylcholine (LysoPC; 20:4, 22:4 and 16:0), and the decrease of anandamide, uric acid and 2,5,7,8-tetramethyl-2(2'-carboxyethyl)-6-hydroxychroman $(\alpha$-CEHC). Among them, 10 metabolites were at higher levels than in the non-CRF group and 11 metabolites were lower than those in the non-CRF group. These data were identified by UPLC/MS and indicated that there were significant differences in the serum metabolic profiles between the CRF and non-CRF groups, which may be used as therapeutic targets for pharmaceutical and non-pharmaceutical interventions in CRF.

\section{Discussion}

In this present study, UHPLC-Q-TOF/MS analysis of blood samples from patients with CRF was performed, aiming to conduct metabolic profiling to identify metabolic biomarkers for CRF. Clear separations between the results of the CRF and non-CRF groups in both positive and negative ion modes of ULPC/MS were observed, which suggested that this method may be further developed to be routinely used for the investigation of the etiology and pathogenesis of CRF.

OPLS-DA identified 21 metabolites with statistically significant differences between the $\mathrm{CRF}$ and non-CRF groups $(\mathrm{P}<0.05)$. Among them, PE $(18: 0 / 0: 0)$ and LysoPE (0:0/20:4 and 0:0/16:0), LysoPC (20:4, 22:4, and 16:0) and the LysoPC/PC ratio, PS (21:0/0:0), glycerophosphocholine and $\mathrm{N}$-docosahexaenoyl $\gamma$-aminobutyric acid increased, and anandamide, uric acid, dihydrouracil, LysoPE (0:0/22:5), $\alpha$-CEHC, 19(R)-hydroxy-prostaglandin F (PGF)1 $\alpha$, ketoleucine, indoxyl sulfate, $\alpha$-N-phenylacetyl-L-glutamine, 1-linoleoyl-glycerophosphocholine and $\mathrm{N}-(3 \alpha, 1-2 \alpha$-dihyd-roxy-5 $\beta$-cholan-24-oyl)glycine decreased.
Due to different structures of lysophosphatides, they have different thermodynamic stability, as well as distinct retention times. When the outer environment changes, lysophosphatides in serum may lead to disease with varying levels of severity. In the present study, multiple serum lysophosphatides, including LysoPE (0:0/20:4 and 0:0/16:0) and lysoPC (20:4, 22:4 and 16:0), increased in patients with CRF. However, other serum lysophosphatides such as LysoPE (0:0/22:5) decreased.

Among these differential metabolites, glycerophospholipids PC, PE and PS represent components of the membrane lipid bilayer, which may be sequentially hydrolyzed by different phospholipases $\left(\mathrm{A}_{1}, \mathrm{~A}_{2}, \mathrm{~B}, \mathrm{C}\right.$ and $\left.\mathrm{D}\right)$. Phospholipase $\mathrm{A}_{2}\left(\mathrm{PLA}_{2}\right)$ hydrolyses phospholipids to lysophospholipids (LysoPC, LysoPE), demonstrating surface activity and is able to cause rupture of cellular membranes, including those of red blood cells, thus causing hemolysis. Lysophospholipids are further hydrolyzed by phospholipase B to glycerophosphocholine and ethanolamine. However, due to the lack of accurate analytical methods to discern this mechanism, little is known about the differential utilization of $\mathrm{PLA}_{2}$ substrates in cells. Furthermore, for biomacromolecules, it is easy to form a series of multi charge ions that may produce a series of multi charge MS peaks, and the charge would increase with the increase of molecular weight, which makes the separation of isotope peaks difficult (17).

Furthermore, there is a lack of methods for the detection of low levels of phospholipids such as PE and PC. These phospholipid substrates are simultaneously used by $\mathrm{PLA}_{2}$ enzymes. Thus, it is important to quantify the PE and PC species within the cell membrane to indicate the content of $\mathrm{PLA}_{2}$ (18). Elevated levels of lysophospholipids in patients with CRF suggest that $\mathrm{PLA}_{2}$ activity may be increased.

It was previously reported that $\mathrm{PLA}_{2}$ activity increases in various pathological conditions, such as inflammation, atherosclerosis and cancer (19), but the role of $\mathrm{PLA}_{2}$ activity in different types of human malignancies has remained controversial (20). A previous study revealed that plasma PLA concentrations increase in patients with different types of cancer. It has been suggested that the inflammation triggered 
Table II. Serum metabolites in patients with CRF and non-CRF subjects.

A, Differential metabolites in positive ion mode.

\begin{tabular}{|c|c|c|c|c|c|c|c|}
\hline Rt (min) & $\mathrm{m} / \mathrm{z}$ & Ion & Formula & Metabolite & Trend & VIP & P-value \\
\hline 0.627299 & 115.051 & {$[\mathrm{M}+\mathrm{H}]^{+}$} & $\mathrm{C}_{4} \mathrm{H}_{6} \mathrm{~N}_{2} \mathrm{O}_{2}$ & Dihydrouracil & $\downarrow$ & 1.563 & 0.011 \\
\hline 0.725687 & 280.092 & {$[\mathrm{M}+\mathrm{Na}]^{+}$} & $\mathrm{C}_{8} \mathrm{H}_{20} \mathrm{NO}_{6} \mathrm{P}$ & Glycerophosphocholine & $\uparrow$ & 1.332 & 0.045 \\
\hline 7.2578 & 414.3 & {$[\mathrm{M}+\mathrm{H}]^{+}$} & $\mathrm{C}_{26} \mathrm{H}_{39} \mathrm{NO}_{3}$ & N-docosahexaenoyl GABA & $\uparrow$ & 3.191 & 0.036 \\
\hline 8.53422 & 502.293 & {$[\mathrm{M}+\mathrm{H}]^{+}$} & $\mathrm{C}_{25} \mathrm{H}_{44} \mathrm{NO}_{7} \mathrm{P}$ & LysoPE (0:0/20:4) & $\uparrow$ & 2.516 & 0.005 \\
\hline 8.99327 & 528.315 & {$[\mathrm{M}+\mathrm{H}]^{+}$} & $\mathrm{C}_{27} \mathrm{H}_{46} \mathrm{NO}_{7} \mathrm{P}$ & LysoPE (0:0/22:5) & $\downarrow$ & 1.869 & 0.009 \\
\hline 9.29651 & 572.371 & {$[\mathrm{M}+\mathrm{H}]^{+}$} & $\mathrm{C}_{30} \mathrm{H}_{54} \mathrm{NO}_{7} \mathrm{P}$ & LysoPC/PC & $\uparrow$ & 1.878 & 0.002 \\
\hline 9.64145 & 301.141 & {$[\mathrm{M}+\mathrm{Na}]^{+}$} & $\mathrm{C}_{16} \mathrm{H}_{22} \mathrm{O}_{4}$ & $\alpha-\mathrm{CEHC}$ & $\downarrow$ & 1.1612 & 0.036 \\
\hline 10.296 & 376.318 & {$[\mathrm{M}+\mathrm{Na}]^{+}$} & $\mathrm{C}_{22} \mathrm{H}_{43} \mathrm{NO}_{2}$ & Anandamide & $\downarrow$ & 1.135 & 0.037 \\
\hline 10.5055 & 373.258 & {$[\mathrm{M}+\mathrm{H}]^{+}$} & $\mathrm{C}_{20} \mathrm{H}_{36} \mathrm{O}_{6}$ & 19(R)-hydroxy-PGF1 $\alpha$ & $\downarrow$ & 1.388 & 0.041 \\
\hline
\end{tabular}

B, Differential metabolites in negative ion mode.

\begin{tabular}{|c|c|c|c|c|c|c|c|}
\hline Rt (min) & $\mathrm{m} / \mathrm{z}$ & Ion & Formula & Metabolite & Trend & VIP & P-value \\
\hline 8.59072 & 588.332 & {$[\mathrm{M}+\mathrm{FA}-\mathrm{H}]^{-}$} & $\mathrm{C}_{28} \mathrm{H}_{50} \mathrm{NO}_{7} \mathrm{P}$ & LysoPC (20:4) & $\uparrow$ & 6.155 & 0.004 \\
\hline 7.25378 & 448.309 & {$[\mathrm{M}+\mathrm{FA}-\mathrm{H}]^{-}$} & $\mathrm{C}_{26} \mathrm{H}_{43} \mathrm{NO}_{5}$ & $\begin{array}{l}\mathrm{N}-(3 \alpha, 12 \alpha \text {-dihydroxy-5} \beta \text {-cholan- } \\
24 \text {-oyl)-glycine }\end{array}$ & & 4.412 & 0.029 \\
\hline 0.784423 & 160.845 & {$[\mathrm{M}-\mathrm{H}]^{-}$} & $\mathrm{C}_{23} \mathrm{H}_{48} \mathrm{NO}_{7} \mathrm{P}$ & PE (18:0/0:0) & $\uparrow$ & 3.848 & 0.000 \\
\hline 8.5608 & 564.331 & {$[\mathrm{M}+\mathrm{FA}-\mathrm{H}]^{-}$} & $\mathrm{C}_{26} \mathrm{H}_{50} \mathrm{NO}_{7} \mathrm{P}$ & 1-Linoleoylglycerophosphocholine & $\downarrow$ & 5.206 & 0.050 \\
\hline 0.973248 & 167.022 & {$[\mathrm{M}-\mathrm{H}]^{-}$} & $\mathrm{C}_{5} \mathrm{H}_{4} \mathrm{~N}_{4} \mathrm{O}_{3}$ & Uric acid & $\downarrow$ & 2.995 & 0.039 \\
\hline 9.1752 & 566.347 & {$[\mathrm{M}-\mathrm{H}]^{-}$} & $\mathrm{C}_{27} \mathrm{H}_{54} \mathrm{NO}_{9} \mathrm{P}$ & PS $(21: 0 / 0: 0)$ & $\uparrow$ & 1.888 & 0.028 \\
\hline 3.82232 & 129.056 & {$[\mathrm{M}-\mathrm{H}]^{-}$} & $\mathrm{C}_{6} \mathrm{H}_{10} \mathrm{O}_{3}$ & Ketoleucine & $\downarrow$ & 2.231 & 0.018 \\
\hline 8.84131 & 452.28 & {$[\mathrm{M}-\mathrm{H}]^{-}$} & $\mathrm{C}_{21} \mathrm{H}_{44} \mathrm{NO}_{7} \mathrm{P}$ & LysoPE (0:0/16:0) & $\uparrow$ & 3.387 & 0.008 \\
\hline 8.89929 & 540.331 & {$[\mathrm{M}+\mathrm{FA}-\mathrm{H}]^{-}$} & $\mathrm{C}_{24} \mathrm{H}_{50} \mathrm{NO}_{7} \mathrm{P}$ & LysoPC (16:0) & $\uparrow$ & 2.182 & 0.016 \\
\hline 3.85521 & 212.003 & {$[\mathrm{M}-\mathrm{H}]^{-}$} & $\mathrm{C}_{8} \mathrm{H}_{7} \mathrm{NO}_{4} \mathrm{~S}$ & Indoxyl sulfate & $\downarrow$ & 1.461 & 0.014 \\
\hline 3.76823 & 263.105 & {$[\mathrm{M}-\mathrm{H}]^{-}$} & $\mathrm{C}_{13} \mathrm{H}_{16} \mathrm{~N}_{2} \mathrm{O}_{4}$ & $\alpha-\mathrm{N}$-phenylacetyl-L-glutamine & $\downarrow$ & 1.363 & 0.015 \\
\hline 9.29567 & 616.363 & {$[\mathrm{M}+\mathrm{FA}-\mathrm{H}]^{-}$} & $\mathrm{C}_{30} \mathrm{H}_{54} \mathrm{NO}_{7} \mathrm{P}$ & LysoPC (22:4) & $\uparrow$ & 4.135 & 0.009 \\
\hline
\end{tabular}

$\uparrow$ and $\downarrow$ respectively indicate the increase and decrease of metabolite content in the CRF group compared to the non-CRF group. CRF, cancer-related fatigue; GABA, $\gamma$-aminobutyric acid; PE, phosphatidylethanolamine; PC, phosphatidylcholine; PS, phosphatidylserine; PGF1 $\alpha$, prostaglandin F1 $\alpha$; M, molecular weight; FA, formic acid; ESI, electron spray ionization; Rt, retention time; m/z, mass-to-charge ratio; VIP, variable importance in the projection.

by tumor cells induces PLA 2 production in a healthy liver, as most cancer cell lines produce proinflammatory cytokines in vitro, such as IL-1 and IL-6. Thus, stimulating liver cells may cause them to produce and release PLA 21 (21). There is also an opinion that PLA $_{2}$ may be produced by cancer cells themselves. The direct and indirect effects of malignant tumors, including disease stress, radiotherapy, chemotherapy and psychological stress, may lead to the increase of reactive oxygen species (ROS) production, which induces increasing lipid peroxidation and PLA 2 activity (22). Elevated levels of lipid peroxidation, $\mathrm{PLA}_{2}$ activity and lysophosphatides may negatively affect the permeability and function of the cell membrane, and it may disrupt the activity of the mitochondria. $\mathrm{PLA}_{2}$ hydrolyzes the Sn-2 fatty acid phospholipid bond of glycerophosphate and then increases the production of lysophospholipids (LysoPC, LysoPE) and free fatty acids. Increased levels of membrane lipid peroxidation, activity of PLA $\mathrm{P}_{2}$ and production of lysophospholipids would lead to the cell membrane being attacked, damage to the membrane phospholipid bilayer, impairment of the cell membrane and mitochondrial membrane fluidity, membrane permeability and impaired enzyme activity. Finally, the physiological function of cells and mitochondria may be abnormal (23).

$\mathrm{PLA}_{2}$ damages membrane lipids, compromises membrane integrity, results in the loss of intracellular creatine kinase, lactate dehydrogenase and haemoglobin, and disrupts oxidative phosphorylation, ATP synthesis and energy metabolism. At the same time, released by lysophosphatides, ROS attacks membrane polyunsaturated fatty acid, which also decreases cell membrane fluidity and compromises oxygen transport and effective microcirculation $(24,25)$. Furthermore, lysophosphatides may cause significant alterations in the shape of red blood cells, thereby converting them to acanthocytes that are subsequently identified and destroyed by the mononuclear phagocytic system and the spleen. Erythrocyte membrane protein thiols are oxidized to disulfides by ROS. 
The oxidized cross-linked hemoglobin is deposited on the inner membrane, leading to increased membrane hardness and changed erythrocyte shape, thereby promoting hemolysis in the microvascular network (26). Chronic hemolysis, abnormal erythrocyte energy metabolism and decrease in mobility would decrease oxygen transport and energy supply to the body's tissues, ultimately causing fatigue symptoms. All of these damages lead to the escape of substances such as creatine kinase and lactate dehydrogenase from the cell (27). These substances are necessary for the oxidative phosphorylation process. Their escape brings the inhibition of the production of ATP and hinders the energy metabolism of red blood cells. Furthermore, histamine, 5-hydroxytryptamine, epinephrine and bradykinin released by tissues after hemolysis would induce inflammation and cause additional inflammatory fatigue (28).

Increased lipid peroxidation, $\mathrm{PLA}_{2}$ activity and lysophosphatide production may affect muscle cell membrane integrity and permeability, thus causing the leakage of intracellular enzymes involved in ATP generation and energy metabolism and the decrease in sodium pump activity and intracellular $\mathrm{K}^{+}$transport (29). Subsequent membrane depolarization and reduction of action potential would result in the decrease of muscle fiber tension and fatigue $(30,31)$.

Energy generation and ATP synthesis in the mitochondria may also be involved. The damage of the mitochondria's inner membrane affects electron transport and oxidative phosphorylation, as well as the control of intracellular $\mathrm{Ca}^{2+}$ transport. Increases of $\mathrm{Ca}^{2+}$ would induce PLA $\mathrm{P}_{2}$ activity, thus creating a vicious cycle in which insufficient ATP synthesis and decreased muscle activity may lead to long-term fatigue (32).

The increase in PE (18:0/0:0) and PS (21:0/0:0), and the phospholipids of the inner membrane, as evidenced by the present study, may lead to the dissociation of phospholipid bilayers and membrane destruction. In addition, the present results revealed an increase in 19(R)-hydroxy-PGF1 $\alpha$, which is a $\omega-1$ hydroxylase metabolite of PGF1 $\alpha$, a derivative of arachidonic acid. Arachidonic acid is an essential fatty acid. It is released by $\mathrm{PLA}_{2}$ and is a precursor in the production of eicosanoids, prostaglandins and leukotrienes, which are associated with tumorigenesis (33). By contrast, the antioxidants uric acid and $\alpha$-CEHC decreased. Uric acid is a free radical scavenger that reduces oxidative stress and lipid peroxidation that is caused by excessive ROS generation $(34,35), \alpha-C E H C$ is a major metabolic product of $\alpha$-tocopherol, which demonstrates antioxidant activity by inhibiting lipid peroxidation (36). The decline in uric acid, $\alpha-\mathrm{CEHC}$, and other antioxidants provides indirect evidence that in patients with $\mathrm{CRF}$, the response to oxidative stress increases.

Regarding changes in the endocannabinoid system, anandamide, also known as arachidonoylethanolamide (AEA), is the earliest discovered endogenous cannabinoid. It is produced from glycerophospholipids by acyltransferase via a phosphodiesterase catalytic reaction. AEA regulates emotions, memory, appetite, the autonomic nervous system and related nervous activities (37-39). Lentiviral-mediated overexpression of fatty acid amide hydrolase reduces the AEA concentration, thus causing anxiety (40). In an animal model of depression, it was proved that depression is associated with the downregulation of cannabinoid-mediated signaling, while the upregulation of endogenous cannabinoid signal transduction may produce antidepressant effects $(41,42)$. In the CRF group, serum AEA decreased, which may be associated with physiological stress-related chronic depression and anxiety characteristics in patients with cancer. Therefore, emotional disorders may be one of the reasons why certain cancer patients feel tired.

The decrease of $\mathrm{N}$ - $(3 \alpha, 12 \alpha$-dihydroxy-5 $\beta$-cholan- 24 oyl)-glycine, $\alpha$-N-phenylacetyl-L-glutamine and other neurotransmitters suggested that CRF may be related to the downregulation of neurotransmission. 5,6-Dihydrouracil (UH2) is a metabolic product of uracil by dihydropyrimidine dehydrogenase (DPD). The UH2/uracil ratio is a marker of DPD activity and may reflect the response of cancer patients to 5-fluorouracil chemotherapy (43). However, the relationship between a decrease in $\mathrm{UH} 2$ and $\mathrm{CRF}$ still requires to be elucidated. The role of changes in other metabolites in CRF should also be further investigated.

In recent years, the development of omics technologies has offered an objective possibility of evaluation, which may be employed for future studies. UPLC/MS is a powerful analytical tool that may be applied to objectively evaluate the biochemical changes and the novel targets associated with CRF (44). The aim of the present study was to research and understand CRF at the metabolic level with the aim of characterizing metabolomic profiles by using the plasma samples from CRF and non-CRF patients (prior to TCM treatment). However, certain limitations that prevent the extension of the present results to more general terms should be noted. First, the number of samples was limited and may be unlikely to identify comprehensive biochemical profiles associated with CRF. As shown in the current results, the different metabolites discovered were limited. It was therefore not possible to proceed to a pathway analysis in the present study. A similar study with a larger sample population may be performed so that a pathway analysis may be possible. Although the sample size in the present study was small, inclusion and exclusion criteria were strict and possible confounding factors that may have influenced metabolic profiles were eliminated. As mentioned above, future research that is based on a larger sample size with database and analytic tools is required to confirm the results of the present study, and pathway investigations should be performed.

The present study identified lysophospholipids (LysoPC and LysoPE) and anandamide as potential biomarkers of $\mathrm{CRF}$, which may be used for the diagnosis of this disorder. Additionally, it suggested that UPLC/MS may be considered as a feasible method to help explore the mechanism of CRF for future studies.

\section{Acknowledgements}

Not applicable.

\section{Funding}

The study was supported by the National Natural Science Foundation of China (grant no. 81673738) and the Three-Year Action Plan for Further Accelerating the Development 
of Traditional Chinese Medicine in Shanghai [grant no. ZY(2018-2020)-FWTX-8007].

\section{Availability of data and materials}

The datasets used and/or analyzed during the current study are available from the corresponding author on reasonable request.

\section{Authors' contributions}

HW and BL conceived and designed the study. TZ and HW acquired the data. HW and BL confirm the authenticity of all the raw data. TZ, CL, ZZ and FF analyzed and interpreted the data. FF, HW and TZ drafted the manuscript. TZ, FF and BL critically revised the manuscript. All authors read and approved the final version of the manuscript.

\section{Ethics approval and consent to participate}

All clinical data were obtained from the 'Research on the efficacy of TCM comprehensive intervention in cancer-related fatigue' (TCM-CRF) project. Medical Ethical Approval for TCM-CRF was approved by the Chinese Ethics Committee of Registering Clinical Trials. The approval number for the TCM-CRF study was ChiECRCT-2013038, and the TCM-CRF study was completed.

\section{Patient consent for publication}

Not applicable.

\section{Competing interests}

The authors declare that they have no competing interests.

\section{References}

1. Haylock PJ and Hart LK: Fatigue in patients receiving localized radiation. Cancer Nursing 2: 461-467, 1979.

2. Wu HS and Harden JK: Symptom burden and quality of life in survivorship: A review of the literature. Cancer Nurs 38: E29-E54, 2015.

3. Arring NM, Barton DL, Brooks T and Zick SM: Integrative therapies for cancer-related fatigue. Cancer J 25: 349-356, 2019.

4. Bussing A, Zhai XF, Peng WB and Ling CQ: Psychosocial and spiritual needs of patients with chronic diseases: Validation of the chinese version of the spiritual needs questionnaire. J Integr Med 11: 106-115, 2013.

5. Rüffer JU, Flechtner H, Tralls P, Josting A, Sieber M, Lathan B and Diehl V; German Hodgkin Lymphoma Study Group: Fatigue in long-term survivors of Hodgkin's lymphoma; a report from the German Hodgkin Lymphoma Study Group (GHSG). Eur J Cancer 39: 2179-2186, 2003.

6. Jim HS, Sutton SK, Jacobsen PB, Martin PJ, Flowers ME and Lee SJ: Risk factors for depression and fatigue among survivors of hematopoietic cell transplantation. Cancer 122: 1290-1297, 2016.

7. Bower JE, Ganz PA, Desmond KA, Bernaards C, Rowland JH, Meyerowitz BE and Belin TR: Fatigue in long-term breast carcinoma survivors: A longitudinal investigation. Cancer 106: 751-758, 2006.

8. Eyob T, Ng T, Chan R and Chan A: Impact of chemotherapy on cancer-related fatigue and cytokines in 1312 patients: A systematic review of quantitative studies. Curr Opin Support Palliat Care 10: 165-179, 2016.

9. Bower JE and Lamkin DM: Inflammation and cancer-related fatigue: Mechanisms, contributing factors, and treatment implications. Brain Behav Immun 30: S48-S57, 2013.
10. Orre IJ, Murison R, Dahl AA, Ueland T, Aukrust P and Fosså SD: Levels of circulating interleukin-1 receptor antagonist and C-reactive protein in long-term survivors of testicular cancer with chronic cancer-related fatigue. Brain Behav Immun 23: 868-874, 2009

11. Schmidt ME, Semik J, Habermann N, Wiskemann J, Ulrich CM and Steindorf K: Cancer-related fatigue shows a stable association with diurnal cortisol dysregulation in breast cancer patients. Brain Behav Immun 52: 98-105, 2016.

12. Cheng S, Shah SH, Corwin EJ, Fiehn O, Fitzgerald RL, Gerszten RE, Illig T, Rhee EP, Srinivas PR, Wang TJ, et al: Potential impact and study considerations of metabolomics in cardiovascular health and disease: A scientific statement from the american heart association. Circ Cardiovasc Genet 10: e000032, 2017.

13. Li N, Liu Y, Li W, Zhou L, Li Q, Wang X and He P: A UPLC/MS-based metabolomics investigation of the protective effect of ginsenosides $\operatorname{Rg} 1$ and $\operatorname{Rg} 2$ in mice with Alzheimer's disease. J Ginseng Res 40: 9-17, 2016.

14. Edge SB and Compton CC: The American Joint Committee on Cancer: The 7th edition of the AJCC cancer staging manual and the future of TNM. Ann Surg Oncol 17: 1471-1474, 2010.

15. Mock V, Atkinson A, Barsevick A, Cella D, Cimprich B, Cleeland C, Donnelly J, Eisenberger MA, Escalante C, Hinds P, et al: NCCN practice guidelines for cancer-related fatigue. Oncology (Williston Park) 14: 151-161, 2000.

16. Piper BF, Dibble SL, Dodd MJ, Weiss MC, Slaughter RE and Paul SM: The revised Piper Fatigue Scale: Psychometric evaluation in women with breast cancer. Oncol Nurs Forum 25: 677-684, 1998 .

17. Gachet MS, Rhyn P, Bosch OG, Quednow BB and Gertsch J: A quantitiative LC-MS/MS method for the measurement of arachidonic acid, prostanoids, endocannabinoids, $\mathrm{N}$-acylethanolamines and steroids in human plasma. J Chromatogr B Analyt Technol Biomed Life Sci 976-977: 6-18, 2015.

18. Stephenson DJ, MacKnight HP, Hoeferlin LA, Park M, Allegood J, Cardona CL and Chalfant CE: A rapid and adaptable lipidomics method for quantitative UPLC-mass spectrometric analysis of phosphatidylethanolamine and phosphatidylcholine in vitro, and in cells. Anal Methods 11: 1765-1776, 2019

19. Murakami M, Taketomi Y, Sato $H$ and Yamamoto K: Secreted phospholipase A2 revisited. J Biochem 150: 233-255, 2011.

20. Dong Q, Patel M, Scott KF, Graham GG, Russell PJ and Sved P: Oncogenic action of phospholipase A2 in prostate cancer. Cancer Lett 240: 9-16, 2006.

21. Yamashita S, Ogawa M, Sakamoto K, Abe T, Arakawa H and Yamashita J: Elevation of serum group II phospholipase A2 levels in patients with advanced cancer. Clin Chim Acta 228: 91-99, 1994.

22. Ruff P, Chasen MR, Long JE and van Rensburg CE: A phase II study of oral clofazimine in unresectable and metastatic hepatocellular carcinoma. Ann Oncol 9: 217-219, 1998.

23. Szlasa W, Zendran I, Zalesińska A, Tarek M and Kulbacka J: Lipid composition of the cancer cell membrane. J Bioenerg Biomembr 52: 321-342, 2020.

24. Bower JE, Ganz PA and Aziz N: Altered cortisol response to psychologic stress in breast cancer survivors with persistent fatigue. Psychosom Med 67: 277-280, 2005.

25. Schaloske RH and Dennis EA: The phospholipase A2 superfamily and its group numbering system. Biochim Biophys Acta 1761: 1246-1259, 2006.

26. Khodadad JK, Waugh RE, Podolski JL, Josephs R and Steck TL: Remodeling the shape of the skeleton in the intact red cell. Biophys J 70: 1036-1044, 1996.

27. Callegari GA, Novaes JS, Neto GR, Dias I, Garrido ND and Dani C: Creatine kinase and lactate dehydrogenase responses after different resistance and aerobic exercise protocols. J Hum Kinet 58: 65-72, 2017.

28. Kolak A, Kamińska M, Wysokińska E, Surdyka D, Kieszko D, Pakieła $M$ and Burdan F: The problem of fatigue in patients suffering from neoplastic disease. Contemp Oncol (Pozn) 21: 131-135, 2017.

29. Enkavi G, Javanainen M, Kulig W, Róg T and Vattulainen I: Multiscale simulations of biological membranes: The challenge to understand biological phenomena in a living substance. Chem Rev 119: 5607-5774, 2019.

30. Theofilidis G, Bogdanis GC, Koutedakis Y and Karatzaferi C: Monitoring exercise-induced muscle fatigue and adaptations: Making sense of popular or emerging indices and biomarkers. Sports (Basel) 6: 153, 2018. 
31. Larsson L, Degens H, Li M, Salviati L, Lee YI, Thompson W, Kirkland JL and Sandri M: Sarcopenia: Aging-related loss of muscle mass and function. Physiol Rev 99: 427-511, 2019.

32. Konig D, Wagner KH, Elmadfa I and Berg A: Exercise and oxidative stress: Significance of antioxidants with reference to inflammatory, muscular, and systemic stress. Exerc Immunol Rev 7: 108-133, 2001.

33. Wang D and Dubois RN: Eicosanoids and cancer. Nat Rev Cancer 10: 181-193, 2010.

34. Ames BN, Cathcart R, Schwiers E and Hochstein P: Uric acid provides an antioxidant defense in humans against oxidant- and radical-caused aging and cancer: A hypothesis. Proc Natl Acad Sci USA 78: 6858-6862, 1981.

35. Yu ZF, Bruce-Keller AJ, Goodman Y and Mattson MP: Uric acid protects neurons against excitotoxic and metabolic insults in cell culture, and against focal ischemic brain injury in vivo. J Neurosci Res 53: 613-625, 1998.

36. Schultz M, Leist M, Elsner A and Brigelius-Flohé R: Alpha-carboxyethyl-6-hydroxychroman as urinary metabolite of vitamin E. Methods Enzymol 282: 297, 1997.

37. Hill MN, Hillard CJ, Bambico FR, Patel S, Gorzalka BB and Gobbi G: The therapeutic potential of the endocannabinoid system for the development of a novel class of antidepressants. Trends Pharmacol Sci 30: 484-493, 2009.

38. Mangieri RA and Piomelli D: Enhancement of endocannabinoid signaling and the pharmacotherapy of depression. Pharmacol Res 56: 360-366, 2007.
39. Lutz B: Endocannabinoid signals in the control of emotion. Curr Opin Pharmacol 9: 46-52, 2009.

40. Rubino T, Guidali C, Vigano D, Realini N, Valenti M, Massi P and Parolaro D: CB1 receptor stimulation in specific brain areas differently modulate anxiety-related behaviour. Neuropharmacology 54: 151-160, 2008.

41. Hill MN, Patel S, Carrier EJ, Rademacher DJ, Ormerod BK, Hillard CJ and Gorzalka BB: Downregulation of endocannabinoid signaling in the hippocampus following chronic unpredictable stress. Neuropsychopharmacology 30: 508-515, 2005.

42. Rossi S, De Chiara V, Musella A, Kusayanagi H, Mataluni G, Bernardi G, Usiello A and Centonze D: Chronic psychoemotional stress impairs cannabinoid-receptor-mediated control of GABA transmission in the striatum. J Neurosci 28: 7284-7292, 2008.

43. Kobuchi S, Ito Y, Okada K, Imoto K, Kuwano S and Takada K: Pharmacokinetic/pharmacodynamic modeling of 5-fluorouracil by using a biomarker to predict tumor growth in a rat model of colorectal cancer. J Pharm Sci 102: 2056-2067, 2013.

44. Hatler CW, Grove C, Strickland S, Barron S and White BD: The effect of completing a surrogacy information and decision-making tool upon admission to an intensive care unit on length of stay and charges. J Clin Ethics 23: 129-138, 2012. 